\title{
WDM Optical Networks: A Complete Design
}

\author{
K. D. R. Assis, W. F. Giozza and H. Waldman, Senior Member IEEE
}

\begin{abstract}
Different integer linear programming (ILP) have been proposed for design of optical networks. The traditional approaches divide design into two separate problems: virtual topology design (VTD), in which best connections among nodes are derived from traffic demand; and routing-and-wavelength assignment (RWA), in which physical paths are accommodated in the physical topology to support the requested connections. We propose an iterative linear programming approach to solve both problems jointly under multiple objectives such as congestion avoidance, fiber load and wavelength pool minimization. The solution of the VTD problem generates a request for a set of paths to be supplied by the physical topology. Physical paths are then allocated in order to minimize some objective functions that are akin to a linear programming formulation. If no feasible solution is found, VTD program supplies a next best solution until all paths are routed. Some objective functions (e.g. maximum fiber load) may be oblivious to the persistence of cycles in the final solution, which may even be dismembered from the source-to-destination link sequence. These anomalies may be eliminated by re-optimizing the solution using the total number of hops as a new objective function, subject to the minimal value of maximum fiber load that was determined in the previous optimization step. The final design phase is the assignment of wavelengths to paths or sections thereof, making best use of available wavelength conversion resources. Our formulation allows for any kind (partial or full, sparse or ubiquitous) of wavelength conversion and limited number of converters, thus providing a tool for the allocation of conversion resources in the network.
\end{abstract}

Index Terms - optical networks; lightpath routing; virtual and physical topology; wavelength conversion.

\section{INTRODUCTION}

$\mathrm{W}$ AVELENGTH Division Multiplexing (WDM) shares the large bandwidth available in optical fibers into multiple channels, each one operating at different wavelengths and at specific data rates (up to 40Gbps). Due to current advances in WDM and high-speed electronic routing/switching, it is likely to be the case that nextgeneration broadband networks will employ a hybrid, layered architecture, using both optical WDM and electronic switching technologies.

\section{A. Optical Networks}

K.D.R Assis is with UFABC/ Center for Engineering, Modelling and Applied Social Sciences UFABC. (corresponding author to provide phone +55114996 3166).

W.F.Giozza is with UNIFACS (corresponding author to provide phone +5571 3330 4630; e-mail:giozza@unifacs.br).

H. Waldman is with UFABC (corresponding author to provide phone +5511 4996 3166; e-mail: waldman@decom.fee.unicamp.br).
The properties of electronic are complementary to those of optics. Electronic processing is ideal for complex nonlinear operations (typical nonlinear operations performed in networks included signal detection, regeneration, buffering, and logic functions), but the limited speed of electronic and optoelectronic devices (e.g., electronic switches, memory devices, processing units, and so on), and the high processing load imposed on electronic in broadband networks, cause the well-know "electronic bottleneck" in optical transmission system. Putting an electronic termination on an optical fiber reduces the potential multiterabit-per-second throughput of the fiber to multigigabit-per-second trickle: the maximum throughput that can expected of the electronics [1], [6].

Although the combination of the ATM technology with the SDH/SONET transport network constitutes a widening of this bottleneck, it is not enough to eliminate it. However, in a WDM networking environment, enabled by optical crossconects [1] and a whole new family of emerging photonic devices [5], the wavelengths may then be alloptically routed to different destinations in the network.

In order to engineer such wavelength coordination, many studies are being accomplished for design of optical WANs, especially in the problem of designing the virtual topology (VTD) to be overlayed on optical networks. The architectural framework assumes transparent clear channels called lightpaths, so named because they traverse several physical links without ever leaving the optical domain from end to end $[2],[5],[6]$.

The question of realization of the virtual topology on an optical infrastructure was set aside temporarily. In the most general setting, optical realization may be include design of the physical (fiber) topology as well as embedding of the virtual topology onto the physical topology. More commonly, the physical topology is given and it is embedding (i.e., lightpath routing and wavelength assignment) [10].

In the design of the physical topology, lightpath routing normally requires that the same wavelength be allocated on all of the links in the path. This requirement is known as the wavelength continuity. The entire bandwidth available on this lightpath is allocated to the connection during its "holding time". The wavelength continuity constraint distinguishes the wavelength routed networks from a traditional circuitswitched network which blocks calls only when there is no capacity along any of the links in the path assigned to the call. Thus, a wavelength-continuos network may suffer from higher blocking as compared to a circuit-switched network.

It is easy to eliminate the wavelength continuity constraints if we are able to convert the data arriving on one wavelength along a link into an other wavelength at an 
intermediate node and forward it along the next link. Such a technique is feasible and is referred to as wavelength conversion.

A wavelength-convertible network which supports complete conversion at all nodes is functionally equivalent to a circuit-switched network, i.e., lightpath requests are blocked only when there is no available capacity on the path. Moreover, in most cases, it may be uneconomic to deploy wavelength conversion capability at all nodes, but having a few nodes with wavelength conversion capabilities may be desirable. Then the question are (1) How many nodes in a network should have conversion capability? (2) How do we choose the converting nodes? (3) How many converters can a node have? (4) How do factors such as traffic demand and network topology affect selection of converting nodes and allocation of converters?

In this paper, we will try to present and discuss the design of virtual and physical topology of optical networks with and without wavelength conversion.

\section{B. Problem Statement}

Although lightpaths underlay SDH/SONET networks in a natural way, packet- and cell- switching client networks, like ATM and IP, would be better served by more packet-oriented WDM layer mechanisms and protocols. However, current optical packet-switching technologies do not yet deliver the same performance that is possible in electronic networks. By looking for the best possible circuit configuration for the traffic demand at any given time, optimizing the virtual topology with constraints of physical topology mitigates the impairments caused by the inability to switch packets on individual basis.

\section{- Virtual topology without resources of the conversion.}

A physical topology is a graph representing the physical interconnection of the wavelength routing nodes by means of fiber-optic cables. In Fig. 1 is show a physical topology of a six-node- wide-area network. The wavelength routing nodes are numbered from 0 to 5 . We consider an edge in the physical topology to represent a pair of fibers, one in each direction.

The set of all unidirectional lightpaths (called $b_{i j}$ 's) set up among the access nodes is the virtual topology. For example, Fig. 2 shows a possible virtual interconnection. There is an edge in the virtual topology between node 2 and node 0 when the data or packets from node 2 to node 0 traverse the optical network in the optical domain only, i.e., undergo no electronic conversion in the intermediate wavelength routing nodes. Edges in a virtual topology are called virtual links.

For example, in Fig. 2 data from node 2 to node 0 are sent on lightpath $b_{20}$ through the wavelength routing node at 1 . Simultaneously, we can send a packet from node 1 to node 3 through the wavelength routing node at 2 . We see that even though in the physical topology there is a fiber connection between node 2 and node 4 , to send a packet from node 2 to node 4 we would have to use two virtual links $b_{20}$ and $b_{04}$. We say that hop length of virtual link $b_{20}$ is two as it traverses two physical edges, $(2,1)$ and $(1,0)$. See [10] for a detailed explanation of this type of routing.

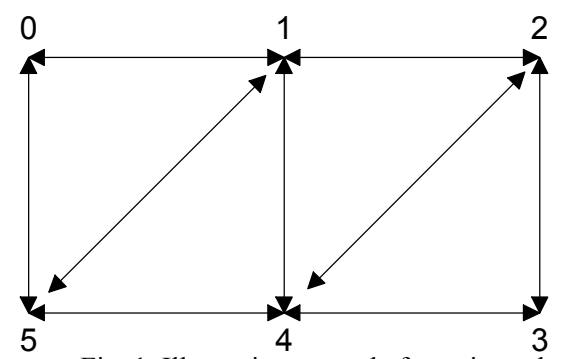

Fig. 1. Illustrative example for a six-node network, physical topology

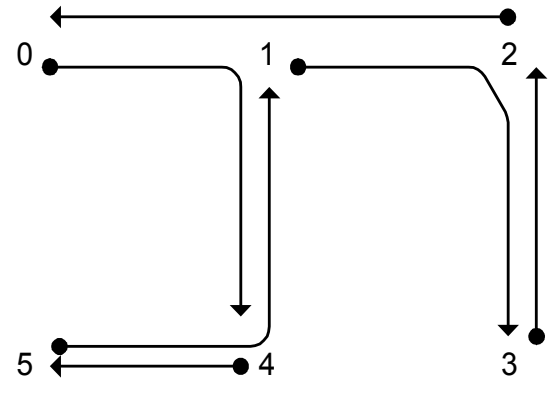

Fig.2. Virtual topology

- Virtual topology with Possible resources of the conversion.

Now, consider a network with six nodes, one edge in physical topology has only one fiber unidirectional and two wavelengths $\left(\varsigma_{1}, \varsigma_{2}\right)$ per fiber, as show Figs. 3 and 4 . The node 0 had resources of the wavelength conversion. Assume that connections are required to be established by lightpaths $b_{25}$, $b_{30}$ and $b_{53}$. The routes that can be used by lightpaths are 2-3$4-5,3-4-5-0$, and 5-0-1-2-3, respectively. In Fig. 4 can be observed that all three connections cannot be established in a network with no converting nodes. If $b_{25}$ uses wavelength $\varsigma_{1}$, then $b_{30}$ cannot use $\varsigma_{1}$, as they share two common links 3-4 and 4-5. Consequently, $b_{30}$ will use wavelength $\varsigma_{2}$. Now, $b_{53}$ cannot use $\varsigma_{l}$, as it is not free on link 2-3. Also, it cannot use $\varsigma_{2}$, as it is not free on link $5-0$. Therefore, $b_{53}$ cannot be established because the route is not wavelength-continuos, even though it is free. The connection $b_{53}$ can be established if node 0 has a converter which can convert from $\varsigma_{1}$ to $\varsigma_{2}$.

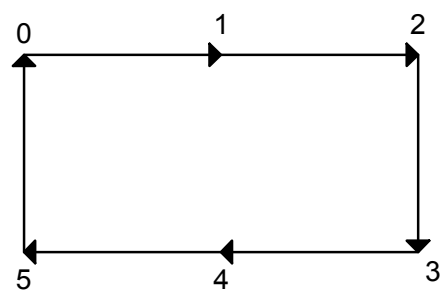

Fig. 3. Unidirectional physical topology 


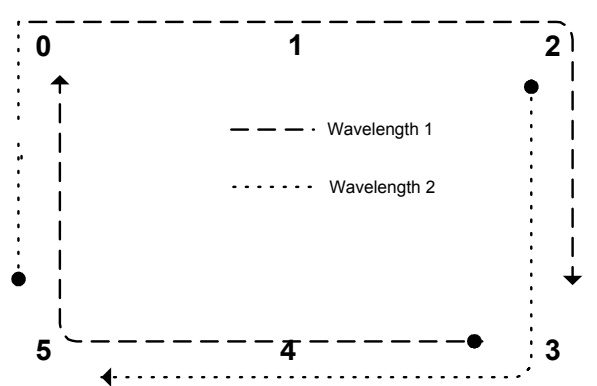

Fig. 4. Virtual topology with resources of the conversion.

It can use $\varsigma_{1}$ on links $5-0$, and $\varsigma_{2}$ on links $0-1,1-2$ and 2-3, as show in figure 4 . It can be noted that a message on this lightpath does wavelength conversion from $\varsigma_{1}$ to $\varsigma_{2}$ at node 0 . This small example shows the advantages of designing a virtual topology with resources of conversion of wavelength.

\section{Objective Functions in Virtual Topology Design}

Different performance measures may be optimized in the design of the virtual topology design and the virtual topology is a solution to an Integer Linear Program (ILP). The following is an enumeration of the some performance measures that form the objective functions for the ILP.

- Traffic congestion on a lightpath is an important parameter as the processing required is proportional to the traffic flowing through a lightpath. Congestion is defined as the maximum traffic that any lightpath in the network carrying. Thus minimizing traffic congestion, the maximum flow (congestion) in a lightpath, may be used as a criterion. This is the objective function in [7].

- The total average hop distance on the virtual topology for lightapths between any sourcedestination pair corresponds to the number of the electro optic conversions for the traffic and is a measure of the delay and also measure of the resources that will be required at the electronic optical bottleneck. This is objective used in [5].

- Minimizing the number of used wavelengths: see $[11,12]$. It is usually assumed in that case that all connections can be established given the available wavelengths and the objective is to use the smallest number of them.

- The maximum number of lightpaths on any physical link is a measure of the expandability of the virtual topology and minimizing it could also be used as an objective in the Integer Linear Program [14].

- Alternatively, if we try to maximize connection in a given session or traffic matrix, for a fixed set of wavelengths, it is called Max-RWA problem. The formulation that solves this problem should maximize the number of lightpaths established using the minimum number of wavelengths [2].

\section{Previous work}

In optical networks to design a virtual topology for a given physical topology, we need to determine the edges (lightpaths) in the virtual topology, choose a route for each of the lightpaths, choose a wavelength for each of the lightpath, and develop a method for routing traffic (still not demonstrated) over the virtual topology. Virtual topology problem can therefore be decomposed into subproblems. Since the virtual topology problem is very complex to solve, the subproblems can be solved individually. Although this results in a suboptimal solution, it is well acceptable for complexity reasons. Some of the existing work solves only a few among of the subproblems, with the assumption that other subproblems have already been solved by some means [15].

In [5] authors formulate the virtual topology design problem as a nonlinear optimization problem. The objective considered was either delay minimization or minimizing the maximum offered load. The same authors subdivide the problem into four subproblems. They are: 1) determining a virtual topology (virtual links); 2) routing the virtual links over physical links; 3) assigning wavelengths to the routes; and 4) routing packet traffic on the virtual topology. The drawbacks of the above approach are the following. 1) If the network is large then using heuristics approach could be computationally very expensive. 2) It is not an integrated approach to solve the four subproblems; rather, it considers subproblems one and four independently. In [6] authors formulated the virtual topology design problem as a linear problem case, the nodes were equipped with wavelength converters. However, in order to cope with wavelength conversion this previous only relax wavelength continuity constraints. The model does not allow to define kinds of conversion. The wavelength continuity constraint of [5] could be introduced in [16], but the "new program" would be harder to solve.

In [7] the problem of virtual topology design is considered but the number of wavelengths the fiber supports is not a constraint. The drawback in this approach is that the physical topology becomes irrelevant for designing a virtual topology.

In [17] is present an exact linear formulation for designing a virtual topology, but with no wavelength converters.

In [2] authors formulated the routing and wavelength assignment problem as a linear program in which all nodes were equipped with partial wavelength changers. Moreover, the model presented at [2] does not allow to define kinds of conversion in each node.

\section{E. Contribution of this work}

We extend the iterative linear programming approach from [13] to solve the subproblems "almost" jointly under multiple objectives such as congestion avoidance, fiber load and wavelength pool minimization with resources of conversion. Our formulation allows for any kind (partial or full, sparse or 
ubiquitous) of wavelength conversion, thus providing a tool for the allocation of conversion resources in the network. To the best of our knowledge, in [14] it was is the first time a linear formulation has been stated which provides a solution to the virtual topology design problem with resources of any kind of conversion and limited number of converters, considering the wavelength continuity constraint. Therefore, in this paper the model from [13] is extended to address an important problem in routed all-optical WDM networks: How to efficiently utilize a limited number of wavelength converters?

\section{F. Outline}

The rest of the paper is organized as follows. In Section II, we show a precise formulation for the VTD and RWA with resources of conversion. Section III explains the heuristic for an integrated virtual and physical topology design. Section IV exemplifies the application on two kinds of network. Section $\mathrm{V}$ shows the extended formulation for limited number of converters per node. Section VI exemplifies the applications and shows some statistics. In Section VII, we propose a heuristic for large networks. Finally, Section VIII presents our conclusions.

\section{FORMULATION OF THE PROBLEM}

We formulate the joint VTD and PTD (Physical Topology Design) problems as an optimization problem. The problem of embedding a desired virtual topology on a given physical topology (fiber network) was formally stated as an exact linear programming formulation.

\section{A. Notation}

- $s$ and $d$ denote source and destination nodes, of the packets or data, respectively.

- $i$ and $j$ denote originating and terminating nodes, respectively, in a lightpath.

- $\quad m$ and $n$ denote endpoints of a physical link that might occur in a lightpath.

B. Given

- Number of nodes in the network: $N$.

- $\quad$ Number of transmitters at node $i: T_{i}\left(T_{\mathrm{i}} \geq 1\right)$. Number of receiver at node $i: R_{i}\left(R_{i} \geq 1\right)$. (same virtual degree)

- Traffic matrix $\Lambda_{s d}$ : is an element of traffic matrix which denotes the average rate of traffic flow from $s$ to node $d$. (normally expressed in bits/second).

- Capacity of each channel: $C$ (normally expressed in bits/second).

- Maximum loading per channel: $\beta, 0<\beta \leq 1$. $\beta$ restricts the queuing delay on a lightpath from getting unbounded by avoiding excessive link congestion.

- Physical Topology $\left(P_{m n}\right)$ : Denotes the number of fibers interconnecting node $m$ and $n . P_{m n}=0$ for nodes which are note physically adjacent to each other. We assume that
$P_{m n}=P_{n m}$, so the number of fibers connecting two nodes in both directions is the same. Note that there may be more than one fiber link connecting adjacent nodes in the network. $\sum_{m n} P_{m n}=M$ denotes the total number of fiber links in the network.

- $\quad$ Number of wavelengths available: $F$

- Set $C_{l}(\varsigma)$ : Set of the wavelengths into which $\varsigma$ can be converted for node $l$.

- Set $D_{l}(\varsigma)$ : Set of the wavelengths that can be converted into $\varsigma$ for node $l$.

\section{Variables}

- Load: $L$ is the maximum load needed in any fiber.

- Lightpath: The variable: $b_{i j}=1$ if there exists a lightpath from node $i$ to node $j$ in the virtual topology; $b_{i j}=0$ otherwise. Note that this formulation is general since lightpaths are not necessarily assumed to be bidirectional, i.e., $b_{i j}=1 \nRightarrow b_{j i}=1$. Moreover, there may be multiple lightpaths between the same source-destination pair, i.e., $b_{i j}>1$, for the case when traffic between nodes $i$ and $j$ is greater than a single lightpath's capacity.

- Traffic routing: The variable $\lambda_{i j}^{s d}$ denotes the amount of traffic flowing from node $s$ to node $d$ and employing $b_{i j}$ as an intermediate virtual link.

- Physical topology route: The variable $p_{m n}^{i j}$ denotes the number of lightpaths between nodes $i$ and $j$ being routed though fiber link $m-n$.

- $\quad c_{i j \varsigma}=$ Number of lightpaths between node $i$ and node $j$ that start in the wavelength $\varsigma$, for $\varsigma=1,2,3, \ldots, W$.

- $\quad d_{i j \varsigma}=$ Number of lightpaths between node $i$ and node $j$ that finish in the wavelength $\varsigma$, for $\varsigma=1,2,3, \ldots, W$.

- Wavelength assignment variables: $p_{m n \varsigma}^{i j}=1$, if the lightpath between node $i$ and $j$ uses wavelength $\varsigma$ through physical link $m-n$.

D. Virtual Topology Design (VTD)

- Objective:

$$
\text { Minimize: } \frac{1}{\sum_{s d} \Lambda_{s d}} \sum_{i j} \sum_{s d} \lambda_{i j}^{s d}
$$

- On virtual topology connection matrix:

$$
\begin{gathered}
\sum_{j} b_{i j} \leq T_{i}, \ldots . \forall_{i} \\
\sum_{i} b_{i j} \leq R_{j}, \ldots . \forall_{j}
\end{gathered}
$$


- On virtual topology traffic variables:

$$
\begin{gathered}
\sum_{j} \lambda_{s j}^{s d}=\Lambda_{s d} \\
\sum_{i} \lambda_{i d}^{s d}=\Lambda_{s d} \\
\sum_{i} \lambda_{i k}^{s d}=\sum_{j} \lambda_{k j}^{s d} \ldots . . i f \ldots . . k \neq s, d \\
0 \leq \lambda_{i j}^{s d} \leq \Lambda_{s d} \cdot\left\{1+\operatorname{sgn}\left(b_{i j}-0,5\right)\right\} / 2 \\
\sum_{s d} \lambda_{i j}^{s d} \leq \beta . C . b_{i j}
\end{gathered}
$$

$$
\text { Int } b_{i j}
$$

\section{E. Physical Topology Design (PTD)}

- E.1) Routing on physical topology $p_{m n}^{i j}$ :

$$
\begin{gathered}
\sum_{m} p_{m k}^{i j}=\sum_{n} p_{k n}^{i j}, \ldots . . i f \ldots . . k \neq i, j \\
\sum_{n} p_{i n}^{i j}=b_{i j} \\
\sum_{m} p_{m j}^{i j}=b_{i j} \\
\sum_{i j} p_{m n}^{i j} \leq L . P_{m n} \quad \forall m, n
\end{gathered}
$$

$$
\text { Int } p_{m n}^{i j}
$$

- $\quad$ E.2) On coloring lightpaths with conversion resources

$$
\begin{gathered}
\sum_{n} p_{i n \varsigma}^{i j}=c_{i j \varsigma} \\
\sum_{m} p_{m j \varsigma}^{i j}=d_{i j \varsigma} \\
\sum_{m} p_{m l \varsigma}^{i j} \leq \sum_{n} \sum_{t \in C_{l}(\varsigma)} p_{\ln t}^{i j}, \quad \text { ifl } l \neq i, j \\
\sum_{n} p_{\ln \varsigma}^{i j} \leq \sum_{m} \sum_{t \in D_{l}(\varsigma)} p_{m l t}^{i j} \quad \text { if } l \neq i, j \\
\sum_{\varsigma} c_{i j \varsigma}=\sum_{\varsigma} d_{i j \varsigma}=b_{i j} \\
\sum_{\varsigma} p_{m n \varsigma}^{i j}=p_{m n}^{i j} \\
\sum_{i j} p_{m n \varsigma}^{i j} \leq P_{m n} \\
\operatorname{Int} p_{m n \varsigma}^{i j}, c_{i j \varsigma}, d_{i j \zeta} .
\end{gathered}
$$

\section{F. Explanation}

In VTD the objective function minimizes the average packet hop distance in the network. The (3.1) is a linear objective function. Eqs. (3.2) and (3.3) ensure that the number of lightpaths emerging from a node is constrained by the number of transmitters at that node, while the number of lightpaths terminating at a node is constrained by the number of receivers at that node. Eqs. (3.4)-(3.6) are multicommodityflow equations governing the flow of traffic through the virtual topology. The (3.7) ensures that traffic can only flow through an existing lightpath, while (3.8) specifies the capacity constraint in the formulation.

In PTD, subsection E.1, (3.9)-(3.11) are multicommodityflow equations governing the routing of lightpaths from source to destination. Eq. (3.12) ensures that the number of lightpaths in a fiber link does not exceed $L$.

In PTD, subsection E.2, (3.13) and (3.14) ensure that a lightpath starts in wavelength $\varsigma$ can be finished in another wavelength. Equations (3.17) guarantee that the number of the lightpath that start at node $i$ is equal to the number of the lightpath that is finished at node $j$, but not necessarily using the same wavelength.

Constraint (3.18) guarantees that the number of wavelengths present in each physical link is equal to the number of lightpaths traversing it.

Constraint (3.19) assured that there is no wavelength clash at any physical link, i.e., no two virtual links traversing through the physical link will be assigned the same wavelength.

Equations (3.15) and (3.16) guarantee that a wavelength that arrives in node $l$ in color $\varsigma$ can be converted for another wavelength in accordance with definition of the $C_{l}(\varsigma)$ and $D_{l}(\varsigma)$ sets. It notices that if it does not have any kind of conversion in node $l$, then $C_{l}(\varsigma)=D_{l}(\varsigma)=\{\varsigma\}$, therefore:

$$
\begin{gathered}
\sum_{m} p_{m l \varsigma}^{i j} \leq \sum_{n} \sum_{t \in C_{l}(\varsigma)} p_{\ln t}^{i j}=\sum_{m} p_{m l \varsigma}^{i j} \leq \sum_{n} p_{\ln \varsigma}^{i j} \\
\text { and } \\
\sum_{n} p_{\ln \varsigma}^{i j} \leq \sum_{m} \sum_{t \in D_{l}(\varsigma)} p_{m l t}^{i j}=\sum_{n} p_{\ln \varsigma}^{i j} \leq \sum_{m} p_{m l \varsigma}^{i j}
\end{gathered}
$$

And these inequalities imply: $\sum_{n} p_{\ln \varsigma}^{i j}=\sum_{m} p_{m l_{\varsigma}}^{i j}$. Therefore, that is a tautology and proves the validity of the sets $C_{l}(\varsigma)$ and $D_{l}(\varsigma)$.

G. Examples:

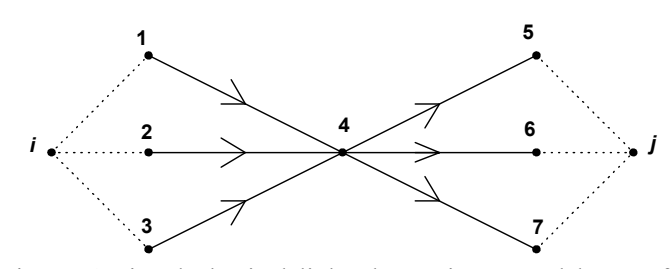

Fig. 5. Optional physical links that arrive to and leave of node 4 for a lightpath from $i$ to $j$. 
For the graph in Fig. 5, the conservation of lightpaths from node $i$ to node $j$ at intermediate node 4 is expressed in the following examples:

- G1) There is no resources of conversion at node 4 . Then, $C_{4}(\varsigma)=D_{4}(\varsigma)=\{\varsigma\}$. W $=\varsigma$, for $\varsigma=\varsigma_{1}, \varsigma_{2}, \varsigma_{3}$.

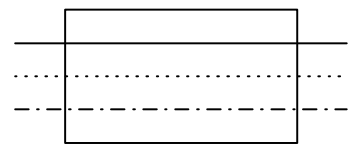

Fig. 6. No conversion for example G1.

Applying 3.15 and 3.16 , we get to:

$$
\begin{aligned}
& p_{14 \varsigma}^{i j}+p_{24 \varsigma}^{i j}+p_{34 \varsigma}^{i j} \leq p_{45 \varsigma}^{i j}+p_{46 \varsigma}^{i j}+p_{47 \varsigma}^{i j} \\
& \text { and } \\
& p_{45 \varsigma}^{i j}+p_{46 \varsigma}^{i j}+p_{47 \varsigma}^{i j} \leq p_{14 \varsigma}^{i j}+p_{24 \varsigma}^{i j}+p_{34 \varsigma}^{i j} \\
& \Rightarrow p_{14 \varsigma}^{i j}+p_{24 \varsigma}^{i j}+p_{34 \varsigma}^{i j}= p_{45 \varsigma}^{i j}+p_{46 \varsigma}^{i j}+p_{47 \varsigma}^{i j} \\
& \Rightarrow \sum p_{\mathrm{ln} \varsigma}^{i j}=\sum p_{m l \zeta}^{i j},
\end{aligned}
$$

- G2) Now, with fixed conversion at node 4, from $\varsigma_{l \rightarrow \varsigma_{2}}$ and $\varsigma_{2 \rightarrow} \rightarrow \varsigma_{l}$, but without any conversion for $\varsigma_{3}$.

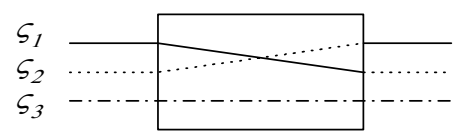

Fig. 7. Types of conversion for example G2.

Then, $\quad C_{4}\left(\varsigma_{1}\right)=\left\{\varsigma_{2}\right\}, \quad D_{4}\left(\varsigma_{1}\right)=\left\{\varsigma_{2}\right\} \quad$ and $\quad C_{4}\left(\varsigma_{2}\right)=\left\{\varsigma_{1}\right\}$, $D_{4}\left(\varsigma_{2}\right)=\left\{\varsigma_{1}\right\}$ and $C_{4}\left(\varsigma_{3}\right)=D_{4}\left(\varsigma_{3}\right)=\left\{\varsigma_{3}\right\}$. Applying these sets for inequalities (restrictions) 3.15 and 3.16, we obtain the following equalities:

$$
\begin{aligned}
& \Rightarrow p_{14 \varsigma 1}^{i j}+p_{24 \varsigma 1}^{i j}+p_{34 \varsigma 1}^{i j}=p_{45 \varsigma 2}^{i j}+p_{46 \varsigma 2}^{i j}+p_{47 \varsigma 2}^{i j} \\
& \Rightarrow p_{14 \varsigma 2}^{i j}+p_{24 \varsigma 2}^{i j}+p_{34 \varsigma 2}^{i j}=p_{45 \varsigma 1}^{i j}+p_{46 \varsigma 1}^{i j}+p_{47 \varsigma 1}^{i j} \\
& \Rightarrow p_{14 \varsigma 3}^{i j}+p_{24 \varsigma 3}^{i j}+p_{34 \varsigma 3}^{i j}=p_{45 \varsigma 3}^{i j}+p_{46 \varsigma 3}^{i j}+p_{47 \varsigma 3}^{i j}
\end{aligned}
$$

- G3) Finally, with a partial conversion at node 4 , as illustrated for the Fig. 8.

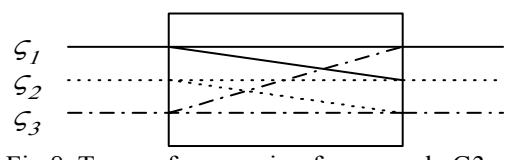

Fig.8. Types of conversion for example G3.

Then, $C_{4}\left(\varsigma_{1}\right)=\left\{\varsigma_{1}, \varsigma_{2}\right\}, D_{4}\left(\varsigma_{1}\right)=\left\{\varsigma_{1}, \varsigma_{3}\right\}$ and $C_{4}\left(\varsigma_{2}\right)=\left\{\varsigma_{2}, \varsigma_{3}\right\}$, $D_{4}\left(\varsigma_{2}\right)=\left\{\varsigma_{1}, \varsigma_{2}\right\}$ and $C_{4}\left(\varsigma_{3}\right)=\left\{\varsigma_{1}, \varsigma_{3}\right\}, D_{4}\left(\varsigma_{3}\right)=\left\{\varsigma_{2}, \varsigma_{3}\right\}$. Applying 3.15 and 3.16, we obtain the following equalities:

$$
\begin{aligned}
\Rightarrow p_{14 \varsigma 1}^{i j}+p_{24 \varsigma 1}^{i j}+p_{34 \varsigma 1}^{i j}= & p_{45 \varsigma 1}^{i j}+p_{46 \varsigma 1}^{i j}+p_{47 \varsigma 1}^{i j} \\
& +p_{45 \varsigma 2}^{i j}+p_{46 \varsigma 2}^{i j}+p_{47 \varsigma 2}^{i j} \\
\Rightarrow p_{14{ }^{\prime j} 2}^{i j}+p_{24 \varsigma 2}^{i j}+p_{34 \varsigma 2}^{i j}= & p_{45 \varsigma 2}^{i j}+p_{46 \varsigma 2}^{i j}+p_{47 \varsigma 2}^{i j} \\
& +p_{45 \varsigma 3}^{i j}+p_{46 \varsigma 3}^{i j}+p_{47 \varsigma 3}^{i j} \\
\Rightarrow p_{14 \varsigma 3}^{i j}+p_{24 \varsigma 3}^{i j}+p_{34 \varsigma 3}^{i j}= & p_{4 \zeta \varsigma 1}^{i j}+p_{46 \varsigma 1}^{i j}+p_{47 \varsigma 1}^{i j} \\
& +p_{45 \varsigma 3}^{i j}+p_{46 \varsigma 3}^{i j}+p_{47 \varsigma 3}^{i j}
\end{aligned}
$$

The previous examples illustrate that the definition of the sets $C_{l}(\varsigma)$ and $D_{l}(\varsigma)$ allows for any kind of the conversion in a network: partial, full, sparse, ubiquitous, etc.

\section{APPROACH For INTEGRATEd Design}

The full problem was decomposed into two subproblems, VTD and PTD, with PTD = Routing and lightpath coloring. The fully integrated problem is solved by the algorithm presented in Fig. 9.

Notice that solving VTD is the same as finding the values of the $b_{i j}$ 's variables. Solving for the routing is the same as finding the values of $p_{m n}^{i j}$ variables, and to decide the coloring of the lightpaths means to find of $p_{m n \varsigma}^{i j}$ variables. If each one, separately, results in viable solutions, the joint problem is solved. Otherwise, if one of subproblems is not feasible, a new virtual topology must be designed and the algorithm is executed again. It is interesting to observe that each one of the problems above must be formulated to optimize a different objective function. The VTD, as expressed in (3.1), was formulated to minimize the average number of virtual hops in the network, which is the same as to maximize the efficiency of resources in the network, as well as to minimize the electronic processing (switching speed) at the nodes. The design of the physical topology has the objective of minimizing $L$, and the lightpath coloring problem aims at minimizing $W$.

The viability of the solutions is characterized by the minimum values of $L$ and $W$. Respectively, to the maximum load of fibers and to the pool of available wavelengths $(F)$.

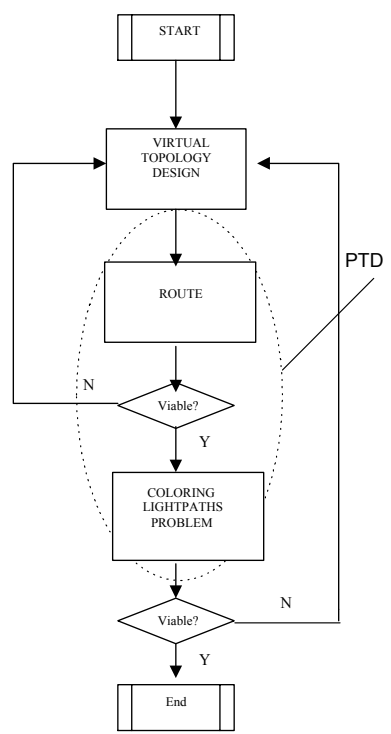

Fig. 9. Heuristic MinW, [13].

If to occur the unfeasibility of the solution of the routing or of the coloring, the rollback to the problem of the virtual topology asks for the next best solution, after the ones that resulted in unfeasible solutions in lower layers. Therefore, the algorithm used for the solution of the VTD must be capable to 
list solutions for $b_{i j}$ 's in the order of their closeness to the optimal value of the objective function.

\section{Simulations I}

For simulation, using the described strategy in the previous section of the following form:

1.) VTD: With the HLDA (Heuristic Topology Design Algorithm), to find the $b_{i j}$ 's for one given matrix of traffic $\Lambda_{s d}$. The heuristic attempts to establish lightpaths between source-destination pairs with the highest $\Lambda_{s d}$ values, subject to constraints of the virtual degree. We find more interesting to decide the VTD as the HLDA, abandoning the equations (3.1)-(3.8), because the HLDA is a classic heuristic [6]. However, the traffic can easily be routing through $b_{i j}$ 's obtained in this step through multicommodity [7].

2.) PTD: The physical topology must ideally be designed with some knowledge about the traffic (lightpaths) that are to be supported over it. Therefore, the next step is to solve (3.9)-(3.12) with a objective function:

$$
\text { Min L }
$$

Our objective is to minimize the maximum load needed in any fiber in the network in order to establish a certain set of lightpaths for a given physical topology and, given $L$, reoptimize (3.9)-(3.19) with a new objective function:

$$
\operatorname{Min} \sum_{m n} \sum_{i j} p_{m n}^{i j}
$$

It is need because maximum fiber load may be oblivious to the persistence of cycles in paths, which may even be dismembered from the source-to-destination link sequence. These anomalies may be eliminated by re-optimizing the solution using the total number of hops as a new objective function. Moreover the coloring is carried through. Therefore we add the equations (3.13)-(3.19). We assume a certain availability of wavelengths and use of available any kind (partial or full, sparse or ubiquitous) wavelength conversion resources in nodes $l$, in accordance with definition of the $C_{l}(\varsigma)$ and $D_{l}(\varsigma)$ sets.

The reoptimization is recommended for multifiber networks, therefore in this in case that we have many options of paths, there is greater possibility of creation of cycles and increment of the number of hops.

The routing and the coloring can be decided integrated. In this case the objective functions explained can be combined into a single function by using a weighting mean $\left(\alpha_{1}\right.$ and $\left.\alpha_{2}\right)$ for normalization:

$$
\operatorname{Min} \alpha_{1} \sum_{m n} \sum_{i j} p_{m n \varsigma}^{i j}+\alpha_{2} . L \text {. }
$$

\section{A. Multifiber Networks}

Multifibers networks are useful when the availability wavelengths is small and the traffic is high. They are also useful for designing survivable networks. In this work we consider that a multifiber network is one with two links unidirectional fibers binding two nodes of the network. That is, it has space division multiplexing. Then, consider the traffic matrix $\Lambda_{s d}$ of Table I, obtained through the generation of random numbers between 0 and 1 with a Gaussian distribution $(\mu=0.5$ e $\sigma=0.1)$. We run the optimization software CPLEX $^{\mathcal{C}}$, and the Table II and III show lightpaths found for virtual degree $1\left(R_{i}=T_{i}=1\right)$ and virtual degree 2 $\left(R_{i}=T_{i}=2\right)$.

For the physical topology of the network of Fig.1 we have $F=3$, with $C_{l}\left(\varsigma_{l}\right)=D_{l}\left(\varsigma_{1}\right)=C_{l}\left(\varsigma_{2}\right)=D_{l}\left(\varsigma_{2}\right)=C_{l}\left(\varsigma_{3}\right)=D_{l}\left(\varsigma_{3}\right)=\left\{\varsigma_{1}, \varsigma_{2}\right.$, $\left.\varsigma_{2}\right\}$, for $\forall l$. That is, it has total conversion in all nodes.

Fig. 10 shows the cycles formed for virtual degree " 1 ". This justifies the re-optimization previously proposal in step 3 . Moreover the shortest path always is the chosen one, for example $b_{34}$ only needs a physical hop after of the reoptimization. These two factors guarantee a minimum number of hop length in the network. Therefore, the success of the reoptimization is guaranteed.

In Fig. 11 the wavelengths had been separate in subnetworks for better visualization. For example, there are two parallels lightpaths from node 0 to node 1 , implying that $b_{011}=1$ and $b_{012}=1$.

TABLE I

\begin{tabular}{c|cccccc}
\multicolumn{7}{|c}{ TRAFFIC MATRIX } \\
\hline$\Lambda_{s d}$ & 0 & 1 & 2 & 3 & 4 & 5 \\
\hline 0 & - & 0,90 & 0,62 & 0,51 & 0,28 & 0,52 \\
1 & 0,53 & - & 0,39 & 0,92 & 0,26 & 0,15 \\
2 & 0,47 & 0,31 & - & 0,34 & 0,21 & 0,14 \\
3 & 0,29 & 0,48 & 0,34 & - & 0,99 & 0,36 \\
4 & 0,15 & 0,44 & 0,14 & 0,84 & - & 0,99 \\
5 & 0,48 & 0,19 & 0,99 & 0,75 & 0,18 & - \\
\hline
\end{tabular}

TABLE II

\begin{tabular}{c|cccccc}
\multicolumn{7}{|c}{ VIRTUAL DEGREE $=1$} \\
\hline$b_{i j}$ & 0 & 1 & 2 & 3 & 4 & 5 \\
\hline 0 & - & 1 & - & - & - & - \\
1 & - & - & - & 1 & - & - \\
2 & 1 & - & - & - & - & - \\
3 & - & - & - & - & 1 & - \\
4 & - & - & - & - & - & 1 \\
5 & - & - & 1 & - & - & - \\
\hline
\end{tabular}

TABLE III

\begin{tabular}{c|cccccc}
\multicolumn{7}{c}{ TABLE III } \\
\hline$b_{i j}$ & 0 & 1 & 2 & 3 & 4 & 5 \\
\hline 0 & - & 2 & - & - & - & - \\
1 & - & - & - & 2 & - & - \\
2 & 2 & - & - & - & - & - \\
3 & - & - & - & - & 2 & - \\
4 & - & - & - & - & - & 2 \\
5 & - & - & 2 & - & - & - \\
\hline
\end{tabular}

In spite of the availability of 3 wavelengths and conversion in all nodes, the solution found did not use all the resources for degree 1 and 2 ( $W$ maximum was 2 and it did not have necessity of conversion in no node). This because in 


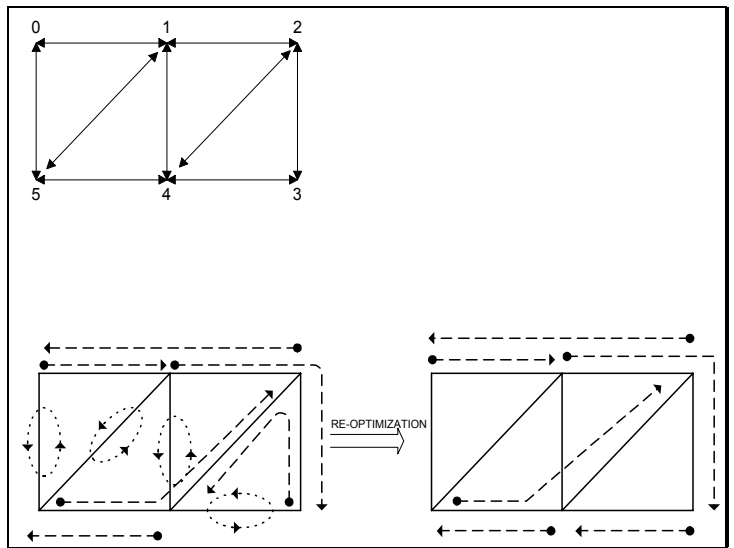

Fig. 10. Re-optimization, for degree virtual "1". $W=1$

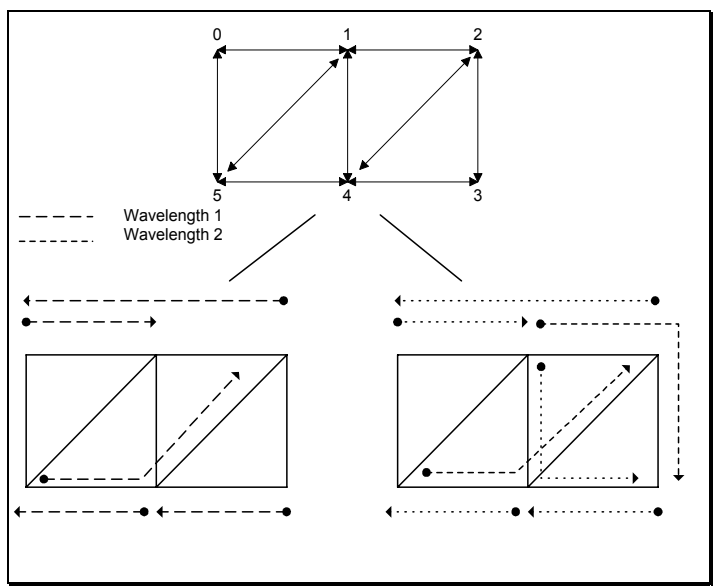

Fig. 11. Degree virtual "2".

this multifiber network more paths can be routed on a link, as there exist multiple copies of wavelengths, one for each fiber on the link. However, the conversion can be necessary for other matrices of connection.

\section{B. Unidirectional fiber networks}

The efficiency of conversion wavelength can be visualized in a unidirectional network (ring), Fig.3. However, all-optical converters are very expensive. Therefore, more practical and cost-effective solution should use only a few converting nodes. In the next simulations $(F=3)$, only one node (each time) of the network will possess conversion (sparse conversion). Then, solutions obtained are compared to the one without any wavelength conversion.

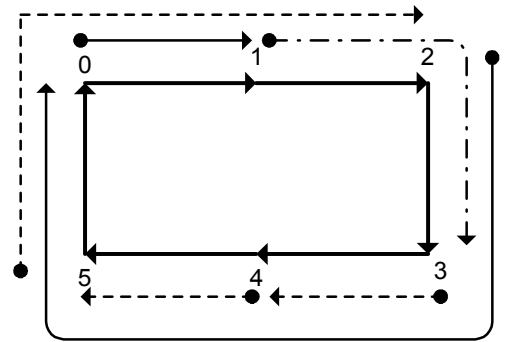

Fig. $\quad$ 12.. $\quad C_{l}\left(\varsigma_{1}\right)=D_{l}\left(\varsigma_{1}\right)=\left\{\varsigma_{l}\right\}, \quad C_{l}\left(\varsigma_{2}\right)=D_{l}\left(\varsigma_{2}\right)=\left\{\varsigma_{2}\right\} \quad$ and $C_{l}\left(\varsigma_{3}\right)=D_{l}\left(\varsigma_{3}\right)=\left\{\varsigma_{3}\right\} \forall l$. "without conversion"

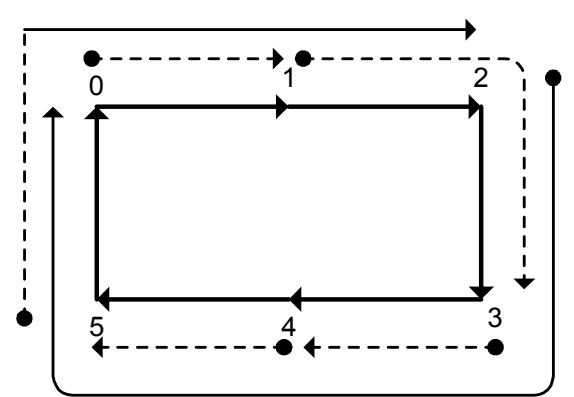

Fig. 13. $C_{l}\left(\varsigma_{1}\right)=D_{l}\left(\varsigma_{l}\right)=\left\{\varsigma_{l}\right\}, C_{l}\left(\varsigma_{2}\right)=D_{l}\left(\varsigma_{2}\right)=\left\{\varsigma_{2}\right\}, C_{l}\left(\varsigma_{3}\right)=D_{l}\left(\varsigma_{3}\right)=\left\{\varsigma_{3}\right\}$, for $l=1,2,3,4,5$ and $C_{l}\left(\varsigma_{1}\right)=D_{l}\left(\varsigma_{l}\right)=C_{l}\left(\varsigma_{2}\right)=D_{l}\left(\varsigma_{2}\right)=C_{l}\left(\varsigma_{3}\right)=D_{l}\left(\varsigma_{3}\right)=\left\{\varsigma_{1}, \varsigma_{2}\right.$, $\left.\varsigma_{2}\right\}$, for $l=0$. "Sparse conversion in 0 "

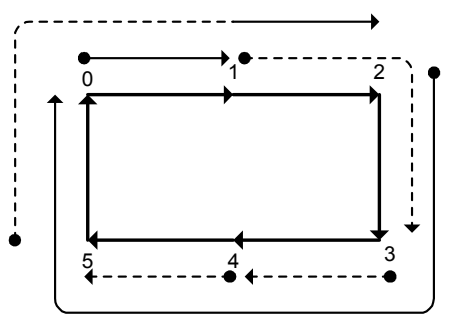

Fig. 14. $C_{l}\left(\varsigma_{l}\right)=D_{l}\left(\varsigma_{l}\right)=\left\{\varsigma_{l}\right\}, C_{l}\left(\varsigma_{2}\right)=D_{l}\left(\varsigma_{2}\right)=\left\{\varsigma_{2}\right\}, C_{l}\left(\varsigma_{3}\right)=D_{l}\left(\varsigma_{3}\right)=\left\{\varsigma_{3}\right\}$, for $l=0,2,3,4,5$ and $C_{l}\left(\varsigma_{l}\right)=D_{l}\left(\varsigma_{1}\right)=C_{l}\left(\varsigma_{2}\right)=D_{l}\left(\varsigma_{2}\right)=C_{l}\left(\varsigma_{3}\right)=D_{l}\left(\varsigma_{3}\right)=\left\{\varsigma_{1}, \varsigma_{2}\right.$, $\left.\varsigma_{2}\right\}$, for $l=1$. "Sparse conversion in 1 "

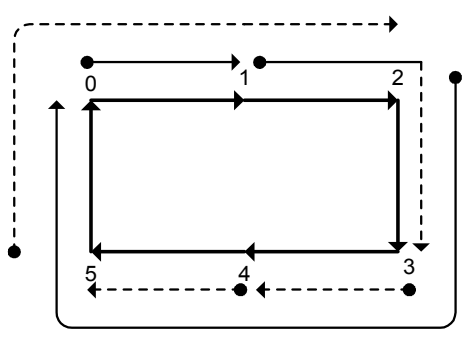

Fig. 15. $C_{l}\left(\varsigma_{1}\right)=D_{l}\left(\varsigma_{1}\right)=\left\{\varsigma_{l}\right\}, C_{l}\left(\varsigma_{2}\right)=D_{l}\left(\varsigma_{2}\right)=\left\{\varsigma_{2}\right\}, C_{l}\left(\varsigma_{3}\right)=D_{l}\left(\varsigma_{3}\right)=\left\{\varsigma_{3}\right\}$, for $l=0,1,3,4,5$ and $C_{l}\left(\varsigma_{l}\right)=D_{l}\left(\varsigma_{1}\right)=C_{l}\left(\varsigma_{2}\right)=D_{l}\left(\varsigma_{2}\right)=C_{l}\left(\varsigma_{3}\right)=D_{l}\left(\varsigma_{3}\right)=\left\{\varsigma_{1}, \varsigma_{2}\right.$, $\left.\varsigma_{2}\right\}$, for $l=2$. "Sparse conversion in 2 "

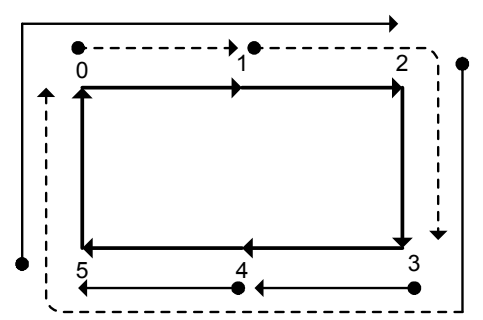

Fig. 16. $C_{l}\left(\varsigma_{1}\right)=D_{l}\left(\varsigma_{1}\right)=\left\{\varsigma_{l}\right\}, l_{l}\left(\varsigma_{2}\right)=D_{l}\left(\varsigma_{2}\right)=\left\{\varsigma_{2}\right\}, C_{l}\left(\varsigma_{3}\right)=D_{l}\left(\varsigma_{3}\right)=\left\{\varsigma_{3}\right\}$, for $l=0,1,2,4,5$ and $C_{l}\left(\varsigma_{l}\right)=D_{l}\left(\varsigma_{l}\right)=C_{l}\left(\varsigma_{2}\right)=D_{l}\left(\varsigma_{2}\right)=C_{l}\left(\varsigma_{3}\right)=D_{l}\left(\varsigma_{3}\right)=\left\{\varsigma_{1}, \varsigma_{2}\right.$, $\left.\varsigma_{2}\right\}$, for $l=3$. "Sparse conversion in 3" 


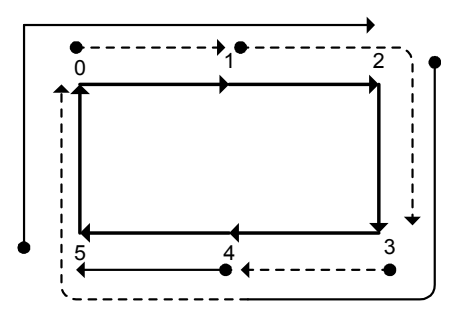

Fig. 17. $C_{l}\left(\varsigma_{1}\right)=D_{l}\left(\varsigma_{1}\right)=\left\{\varsigma_{l}\right\}, C_{l}\left(\varsigma_{2}\right)=D_{l}\left(\varsigma_{2}\right)=\left\{\varsigma_{2}\right\}, C_{l}\left(\varsigma_{3}\right)=D_{l}\left(\varsigma_{3}\right)=\left\{\varsigma_{3}\right\}$, for $l=0,1,2,3,5$ and $C_{l}\left(\varsigma_{l}\right)=D_{l}\left(\varsigma_{l}\right)=C_{l}\left(\varsigma_{2}\right)=D_{l}\left(\varsigma_{2}\right)=C_{l}\left(\varsigma_{3}\right)=D_{l}\left(\varsigma_{3}\right)=\left\{\varsigma_{l}\right.$, $\left.\varsigma_{2}, \varsigma_{2}\right\}$, for $l=4$. "Sparse conversion in 4"

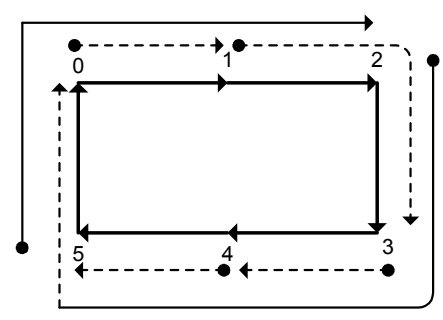

Fig. 18. $C_{l}\left(\varsigma_{l}\right)=D_{l}\left(\varsigma_{l}\right)=\left\{\varsigma_{l}\right\}, C_{l}\left(\varsigma_{2}\right)=D_{l}\left(\varsigma_{2}\right)=\left\{\varsigma_{2}\right\}, C_{l}\left(\varsigma_{3}\right)=D_{l}\left(\varsigma_{3}\right)=\left\{\varsigma_{3}\right\}$, for $l=0,1,2,3,4$ and $C_{l}\left(\varsigma_{1}\right)=D_{l}\left(\varsigma_{l}\right)=C_{l}\left(\varsigma_{2}\right)=D_{l}\left(\varsigma_{2}\right)=C_{l}\left(\varsigma_{3}\right)=D_{l}\left(\varsigma_{3}\right)=\left\{\varsigma_{1}, \varsigma_{2}\right.$, $\left.\varsigma_{2}\right\}$, for $l=5$. "Sparse conversion in 5 "

\section{Notes:}

- One can observe that Fig.12 needed 3 wavelengths while that the others (Figs.13-18) only of 2. That is, without any type of conversion and $\mathrm{W}=2$ the network would suffer blocked.

- The availability of converters in all nodes (ubiquitous) is not necessary. That is, the reduction of pool of wavelengths is found with sparse conversion.

- Beyond sparse conversion the chosen node could use partial conversion of wavelength instead of total conversion. This would not change the final configuration.

\section{LIMTED NUMBER OF CONVERTERS}

In this section we focus on question (3) from introduction. In order to provide an answer, the key point is the formulation of the physical topology design (subsection II-E) problem in networks with small numbers of converters per node. While previous section and literature considers conversion with unlimited or large number of converters in nodes [3], [8], our strategy considers modifications in RWA formulation to allow allocation of a limited number of converters in a node of the network. It is important because this number, if small, is cost effective.

In Fig. 19, in order to allow the specification of a limited number of converters, a node with resources of conversion is split into two auxiliary nodes $a$ and $b$. After that, it is created one unidirectional arc $a-b$. If we would like to have one converter, it is created one more auxiliary node $c_{l}$ and two more auxiliary arcs $a-c_{1}$ and $c_{1}-b$. If we would like to have two converters, it is created one more auxiliary node $c_{2}$ and two more auxiliary arcs $a-c_{2}$ and $c_{2}-b$ and so on. Note that in auxiliary graph constraints arcs "from" or "to" $c_{i}$ have load $L=1$ and the arc from $a$ to $b$ don't have traditional clash constraints. Therefore (3.12) and (3.19) must be replaced by two other equations to guarantee the success of the strategy. These two new equations will be shown in the next subsection.

\section{A. Auxiliar Graph (formulation)}

In Fig. 19, $a, b$ and $c_{i}$ denote nodes from the auxiliary graph (for established number of converters $N_{c}$ with $i=1,2 \ldots . N_{c}$ ). Therefore, if the auxiliary graph is constructed, i.e., there should be established limit for number of converters. Thus, we replace constraints (3.12) and (3.19) by the following more restrictive equations and in addition, we define the $N_{c}$.

$$
\begin{aligned}
& \sum_{i j} p_{m n}^{i j} \leq\left\{\begin{array}{cc}
L . P_{m n} & \text { for }(\operatorname{link} m-n) \neq\left(\operatorname{link} a-c_{i}\right) \\
& \text { or } \neq\left(\operatorname{link} c_{i}-b\right) \\
1 & \text { for }(\operatorname{link} m-n)=\left(\operatorname{link} a-c_{i}\right) \\
& \text { or }=\left(\operatorname{link} c_{i}-b\right)
\end{array}\right. \\
& \sum_{i j} p_{m n \varsigma}^{i j} \leq P_{m n} \quad \text { for }(\text { link } m-n) \neq(\text { link } a-b)
\end{aligned}
$$

\section{Notes:}

- It was observe in the previous section, the number $N_{c}$ is not considered in formulation. Therefore it can be ilimited when there are conversion resources in one or more nodes.

\section{B. Complexity}

In the formulation with auxiliary graph, based in the strategy of Fig. 19, with sparse conversion in one node, the number of nodes grows from $N$ to $N+2$, for 1 converter. From $N$ to $N+3$ for 2 converters and so on. Therefore, the number of nodes grows from $N$ to $N+N_{c}+1$ nodes, when $N_{c}$ converters are put in one node of the network. Similarly, the number of links $E$ grows from $E$ to $E+3$, for 1 converter, from $E$ to $E+5$ for 2 converters and so on. Therefore the number of links grows from $E$ to $E+1+2 . N_{c}$ when $N_{c}$ converters are put in one node of the network. If the same number of converters are put in all $N$ nodes of network, then the number of nodes in formulation grows from $N$ to $2 N+\left(N . N_{c}\right)$ and the number of links grow from $E$ to $E+\left(1+2 . N_{c}\right) . N$. Issues, like the number of routing variables $O\left(N^{2} . E . W\right)$, where there are $W$ wavelengths, will become critical in analyzing scalability. However, in the network practical design the cost is minimized when the minimum number of converters is used, because the node design will be carried through with little equipments [8].

In [19] authors propose an ingress edge node architecture with fixed wavelength converters that have limited wavelength convertibility but are more economical than full wavelength converters. In architecture, each input access link of ingress edge nodes is equipped with fixed wavelength converters, and input wavelengths from the access links are evenly distributed on the output core link. As a result, competition for a free wavelength on an output core link is avoided. Simulation results show that the architecture offers about $20 \%$ cost reduction compared with a node architecture that uses only full wavelength converters. 


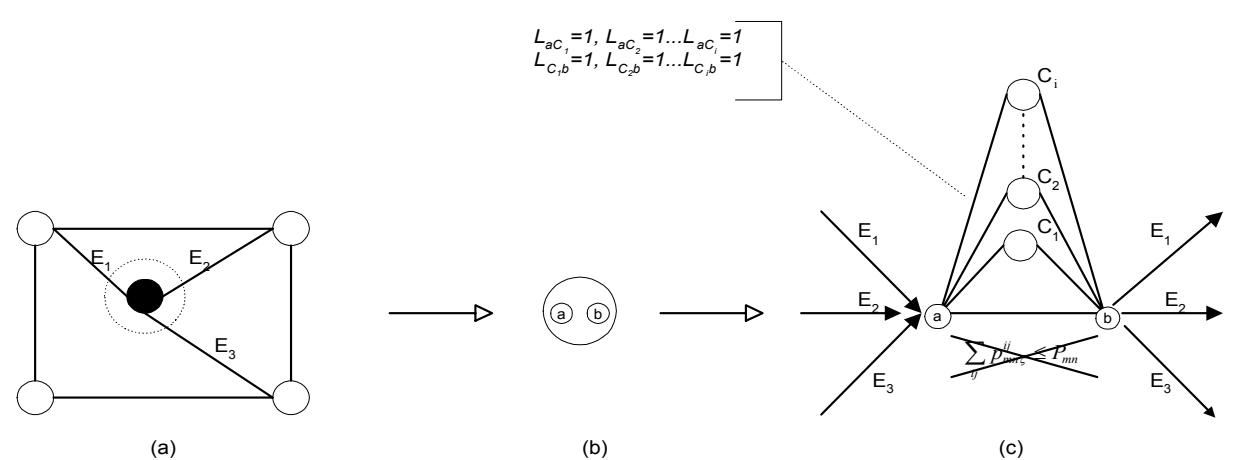

Fig.19. a) The original graph with black nodes, b) Black node is splitting in two nodes ( $a$ and $b$ ) c) A auxiliary graph with auxiliary nodes $a, b$ and $c_{i}$; $a$ is incoming of arcs, $b$ is out of arcs and $c_{i}$ is an auxiliary node

\section{Simulations II}

Some research work in the literature assume that the virtual topology is known already. The source-destination pairs between which a certain number of lightpaths are required to be established are taken as inputs. Such pairs might have been obtained by considering traffic requirements. The PTD is considered only to solve the rest of the subproblems (routing and wavelength assignment). In this section, the problem of establishing lightpaths for a static traffic demand has been considered more one time. The traffic demand is assumed to be static, that is, the set of lightpaths are specified in terms of their physical routes. Therefore, this section assumes that VTD have been solved. Our objective is to maximize the number of lightpaths to be established from the traffic matrix. That is, $K_{i j}$ (is an element of traffic matrix which denotes the number of lightpaths flow from $i$ to node $j$.) is data and the new objective function will be:

$$
\operatorname{Max} \sum_{i j} b_{i j}
$$

With an additional constraint: $\quad b_{i j}<=K_{i j}$

\section{A. The simple example}

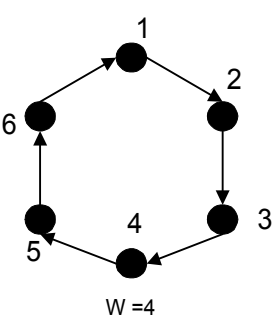

(a)

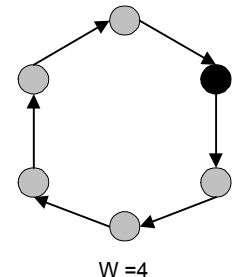

(b) $\begin{array}{lllllllllll}0 & 2 & 0 & 0 & 0 & 0\end{array}$

$2 \begin{array}{llllll}2 & 0 & 0 & 2 & 0 & 0\end{array}$

$\begin{array}{lllllllllll}0 & 0 & 0 & 0 & 0 & 0\end{array}$

$\begin{array}{lllllllllllllll}0 & 0 & 0 & 0 & 2 & 0\end{array}$

$\begin{array}{llllll}0 & 0 & 0 & 0 & 0 & 2\end{array}$

$\begin{array}{llllll}0 & 0 & 2 & 0 & 0 & 0\end{array}$

(c)
Fig. 20. (a) $N_{r}=6$ and (b) $N_{r}=1$ : two unidirectional rings (clockwise direction). Black nodes-conversion. Clear nodes-no conversion, (c) Traffic matrix (12 connections).
TABLE IV

COMPUTATIONAL COST FOR AUXILIARY GRAPH

\begin{tabular}{|c|c|c|c|c|}
\hline \multicolumn{5}{|c|}{ COMPUTATIONAL COST FOR AUXILIARY GRAPH } \\
\hline \multirow{3}{*}{ (a) $N r=6$} & $N_{c}$ & Node cost & Link cost & Connection \\
\cline { 2 - 5 } & 1 & 18 & 24 & 12 \\
\cline { 2 - 5 } & 2 & 24 & 36 & 12 \\
\cline { 2 - 5 } & 3 & 30 & 48 & 12 \\
\hline (b) $N r=1$ & 1 & 36 & 60 & 12 \\
\cline { 2 - 5 } & 2 & 8 & 9 & 12 \\
\cline { 2 - 5 } & 3 & 9 & 11 & 12 \\
\hline & 4 & 11 & 15 & 12 \\
\hline
\end{tabular}

Notice that with the application in formulation/strategy with $N_{c}=2$ in one node, Fig. 20, we will obtain 12 connections, what proves the efficiency and cost saving of the formulation. Notice in Table IV, that in auxiliary graph, the computational cost increases, see previous subsection, and is given by: Node cost $=N+N_{r} \cdot\left(1+N_{c}\right)$, Link cost: $=E+N_{r} \cdot(1+2$. $N_{c}$ ), where $N r$ is the number of nodes with conversion resources.

\section{B. Trivial Heuristc}

An exhaustive approach that enumerates all the possible ways of converter number placement and choosing the best one is not efficient for large networks. In this subsection, we propose simulations in the following way:

1.To allow full conversion in all nodes of the network in the original graph, to apply the traditional formulation and to get the maximum number of established connections. This way, the RWA suppose that all node has unlimited or large number of converters in each node of the network, in agreement with the physical-outdegree of this node. For example, in a node with physical-out-degree $\delta p$ and $\mathrm{W}$ wavelengths, the number of converters $N_{c}$ will allow $N_{c}=W . \delta p$ (as it is applied for all nodes, we have ubiquitous conversion). This step serves only for comparison.

2.Using the auxiliary graph (proposed strategy), to place $N_{c}=1$ in all nodes of the network. To apply the modified formulation and to verify the established number of connections. 


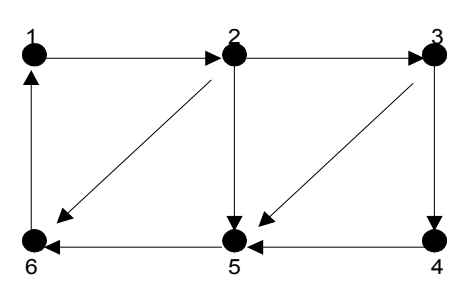

(a)
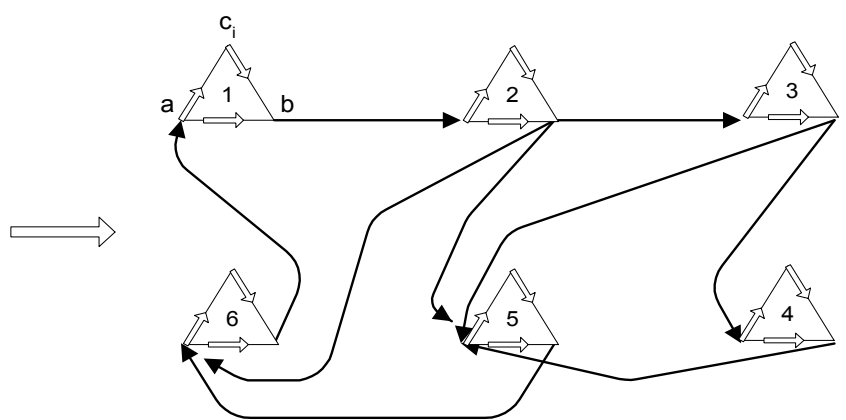

(b)

Fig. 21. (a) 6 node-mesh topology (b) auxiliary graph for 6 node-mesh topology.

3.If the number of established connections in previous step is not same the number obtained in step one, return to the auxiliary graph (step 2) with $N_{c}=2$, and so on.

Notice that when we use the auxiliary graph, the connections always have their origin in auxiliary nodes " $b$ " and always finish in auxiliary nodes "a". For example (Fig. 21b), connections that are initiated in node " 1 " in the original graph, are initiated in the node "b" in auxiliary graph and connections which are finished in node " 1 " in original graph, are finished in the node "a" in auxiliary graph.

\section{Numerical Results for 6 node-mesh topology}

Simulations have been carried out to investigate the performance of the proposed strategy with auxiliary graph over an 6-node mesh topology (Fig.21).

The traffic matrix used by this network is shown in Table $\mathrm{V}$. The total number of connections to be set up is 29 .

\section{TABLE $\mathrm{V}$}

Traffic matrix for 6 node-mesh network

$\begin{array}{llllll}0 & 0 & 0 & 0 & 0 & 0 \\ 0 & 0 & 3 & 3 & 0 & 7 \\ 0 & 0 & 0 & 0 & 7 & 0 \\ 0 & 0 & 0 & 0 & 3 & 0 \\ 3 & 0 & 0 & 0 & 0 & 0 \\ 0 & 0 & 0 & 0 & 3 & 0\end{array}$

The results with linear program are tabulated in Table VI for the cases of No conversion/original graph. Full conversion with $N_{c}=1$ /auxiliary graph and Sparse conversion/auxiliary graph with one or two nodes with $N_{c}=1$.

In table VI, the number of connections obtained with $N c=$ 1 in all nodes is same to the number obtained with ubiquitous conversion with $N c$ unlimited, for all wavelength plans; therefore, the latter is not shown and the step 2 is run only one time for each plan. However, a more effective strategy is to put converters only in one/some (sparse conversion) nodes of the network; in this case the placement order of converters is the following: first, one converter is put on node 1; if the number of established connections is less than in the case of ubiquitous conversion with $N c=1$, then another converter is put on node 2, and so on. In table VI with sparse conversion, for $W=2, W=3$ and $W=6$ with only one converter in node 1 we will establish the same number of connections from $N c=1$ in all nodes, and for $W=4$ and $W=5$ with 2 converters: one converter in node 1 and one converter in node 2 we will also establish the number of the connections from $N c=1$ in all nodes. However, for $W=4$ and $W=5$, if we just one converter in node 1, we will establish 21 and 26 connections, respectively; so the total number of connections can not be established. Therefore the strategy proposed can be used to guide the placement of converters at the design of a network with RWA using the minimum number of converters, as seen in the Fig. 22.

\section{Some comments}

Integer linear programming are popular in the literature as they provide formal descriptions of the problem. In practice, however, scalability to networks with at least tens of nodes, with hundreds of demands is required. In many cases all but trivial instances of theses ILP's are computationally difficult with current state-of-the-art software. The complexity of our formulation grows as demonstrated in sub-section (V.b). In our 6-node mesh network, on average our strategy run be the optimization software CPLEX ${ }^{\odot}$ took around five seconds on an Intel Pentium IV/1.6Ghz. However, in a large network as the NSFNET, with matrix of traffic given for [7], and $N_{c}=1$ for all nodes, our strategy exceeded the CPU memory restriction. Thus, in next subsection a heuristic is developed to find solutions to problems with size typically found in practice.

\section{K SHORTEST PATH HEURISTIC WITH LIMITED NUMBER OF CONVERTERS (KSPNC)}

Here we present a computationally less intensive heuristic algorithm. We propose an experimental comparison of the heuristic from [2], the KSPH, with a $K$ shortest path heuristic for solving the Max-RWA problem with limited number of converters, KSPNc.

Assuming that in the lightpath request matrix, the largest lightpath request between any source-destination pair is $\mathrm{m}$, we find the $K$ shortest paths, wherein $K$ is greater than $m$. The algorithm proceeds in following steps: 
Step 1: Finding $K$ shortest paths in terms of hop-length between all source-destination pairs in traffic matrix:

The $K$ shortest paths are store in lightpaths1, lightpaths $2, \ldots$, lightpaths $K$ arrays. Consider the first shortest path array i.e., lightpaths 1 for processing and go to Step2.

Step 2: Wavelength assignment to the lightpaths:

For the lightpath which is not wavelength assigned in the traffic matrix, choose the path for that from the chosen $\mathrm{K}$ shortest path array. A typical lightpath between nodes (1) and (N) is represented as node [1], node $[2], \ldots$, node $[Q], \ldots$, node $[N]$; where nodes node $[2], \ldots$, node [Q] are nodes along the lightpath. The physical fiber links of the lightpath (node [1], node [2]) labeled as link 1, (node [2], node [3]) as link 2 and the last link (node[N-1], node [N]) as link $N-1$. The first physical link of the lightpath (here node [1], node [2]) is taken and scanned for a free wavelength. If a wavelength $\varsigma_{j}$ is free, then we try to find in all links of that lightpath for the availability of the wavelength $\varsigma_{j}$. Then the algorithm proceeds further, differently for the following cases:

For the case of no conversion of wavelength along the lightpath, if the wavelength $\varsigma_{j}$ is available in all physical links along the lightpath, then we allocate that wavelength for the lighpath. If the continuity of the wavelength $\varsigma_{j}$ along the links in the ligthpath is not possible then, we scan for the next free wavelength $\varsigma_{j}$ on the link (node [1],node [2]). As before the availability of the wavelength $\varsigma_{j}$ on all the physical links on the ligthpath is checked, if the wavelength is available then it is assigned, else we scan for the next free wavelength on the link (node [1], node[2]) and the above procedure for wavelength assignment is repeated till the lightpath is wavelength assigned to the wavelength in the link (node[1], node [2]) is exhausted.

For the case of limited wavelength conversion, if wavelength $\varsigma_{j}$ is blocked on any link" $n$ ", then go back one physical link towards the source node of the lighpath " $n-1$ " and try to obtain a free wavelength by wavelength conversion. If wavelength conversion is not available go back further one link " $n-2$ " and try to obtain a free wavelength by wavelength conversion. Repeat the above procedure till a free wavelength is obtained or link 2 is reched on the lightpath. If a free wavelength is not available at link 2 then go to link 1 and choose a new free wavelength and traverse the physical links of the lightpath towards the destination node assigning wavelengths with or without conversion. While back tracking for a free wavelength if at any intermediate link if we get a free wavelength after conversion, then traverse from that link towards the destination node assigning wavelength with or without conversion. The wavelength conversion allowed at any node depends on the degree of conversion allowed and number of converters $N_{c}$ allowed.

Step 3: Repeat the step 2 till all the lightpaths in the chosen array are exhausted.

Step 4: If any of the lightpaths in given traffic matrix is not wavelength assigned, then choose the next of the $K$ shortest path arrays and go to step 2. If all the lightpaths are wavelength assigned or all the $K$ shortest path arrays are exausted, then stop the algorithm.

\section{B. Numerical Results}

The NSFNET shown in Fig. 23 is a 14 node network with 21 edges. In this network each edge represents a pair of fibers, one in each direction.

The traffic matrix that has to be realized over the NSFNET is shown in the table VII. We assumed that at most 3 multiple connections were permitted for a source-destination pair. The number of connections are chosen from $0,1,2,3$ with equal probability for a source destination pair. The total number of connections to be set up is 268 . For comparison with [2], we assumed:

All nodes in the network are equipped with wavelength converters with limited conversion capability. Therefore, only the case "ii" in step 2 from heuristic is considered.

The degree of conversion is 3 . For help the reader, see Fig.8, it the degree of conversion is 2 .

First, we relax the integer constraints of mathematical formulation, as [2]. For a given wavelength, we find LP upper bound.

After, we executed the KPSH and KPSNc algorithm on the NSFNET network with the value of $K=5$. The greater the value of $K$, more will be number of connections realized, because there are more alternative paths available for wavelength assignment. The KPSNc results based on the number of converters are captured in table along with results of LP (upper bound) and KPSH Heuristic with large $N_{c}$ from [2].

Comparing in table VIII, we find that in terms of performance (Established Connections); LP (upper bound), KPSH, KPSNc with $N_{c}=7$ and KPSNc with $N c=5$ gives better performance in that order. In LP, this happens because of the relaxed integer constraints. In $\mathrm{KSPH}$, this happens because of large number of converters. The KSPNc had limited number of converters, therefore the performance is less or the same from $W=10$ to $W=20$. However, in the network practical design the cost is minimized when the minimum number of converters is used. Then, KSPNc is a heuristic cost-effective.

\section{CONCLUSIONS}

In this paper we have looked at design issues in optical networks with and without wavelength conversion. We have proposed mathematical formulation and algorithms for solution to the virtual and physical topology design of optical networks.

We have characterized the solution to the RWA problem with allocation number of converters and kinds of conversion.

We have proposed a $K$-shortest path heuristic approach for limited number of converters and kinds of conversion for large optical networks.

The formulation/strategy proposed in this paper have a significant impact to the well-known RWA problem. First, it can help understand the relationship between the number of wavelengths required and the number of converters. Second, it can be used to guide the placement of converters at the design 
of a network. Third, a new feature of the proposed formulation is that any kind of conversion can be made in each node of the network. This is obtained by using more general constraints.

By recently developed heuristic technique advancements [18], the best known solution could be improved for more instances with minimum converter wavelength assignment, again proving optimality. Besides further founding the benefit of our approach, this observation also indicates that the heuristics algorithms are still improvable.

\section{ACKNOWLEDGMENTS}

This work was supported by FAPESP, FAPESB, CNPq and by the Research and Development Center, Ericsson Telecomunicações S.A., Brazil.

\section{REFERENCES}

[1] R. Ramaswami and K.N. Sivarajan, "Optical Networks: A Practical Perspective", Morgan Kaumann Publishers, ISBN 1-55860-445-6, San Francisco, USA,1998.

[2] M.D. Swaminathan and K.N Sivarajan, "Practical Routing and Wavelength Assignment algorithms for All Optical networks with Limited Wavelength Conversion" ICC 2002.

[3] R. Ramaswamy and G. Sasaki, "Multiwavelength Optical Networks with Limited Wavelength Conversion", IEEE/ACM Transactions on Networking Vol. 6. No. 6. December 1998.

[4] Byrav Ramamurthy and Biswanath Mukherjee, "Wavelength Conversion in WDM Networking", IEEE Journal on Selected Areas in Communication (1998) vol 16, No 7, pp 1061-1073.

[5]D. Banerjee and B. Mukherjee. "Pratical approaches for routing and wavelength assignment in large all-optical wavelength-routed networks." IEEE Journal on Selected Areas in Communications, 14 (5): 903-908, June 1996.

[6] C. Siva Ram Murthy and Mohan Gurusamy “WDM Optical Networks: Concepts, Design, and Algorithms. Prentice Hall, 2002. ISBN 0-13060637-5.

[7] R. Ramaswami and K.N.Sivarajan, "Design of logical topologies for wavelength-Routed All Optical Networks", IEEE/JSAV, vol. 14, pp. 840-851, june 1996.

[8] Yiming Z. and Oliver W.W. Y “ An Effective approach to the connection routing problem of all-optical wavelength routing DWDM networks with wavelength conversion capability" ICC 2003.

[9] R. Ramaswami and K. N. Sivarajan, "Routing and wavelength assignment in all-optical networks", IEEE/ACM Trans. Networking, vol. 3, n.5, pp. 489-500, Oct. 1995

[10]H. Zang, J. P. Jue, and B. Mukherjee, “A Review of Routing and Wavelength Assignment Approaches for Wavelength-Routed Optical WDM Networks," Optical Networks Magazine, vol. 1, no. 1, pp. 47-60, Jan. 2000.

[11]K. Lee, K.Kang , T. Lee, and S. Park. An optimization approach to routing and wavelength assignment in WDM all-optical mesh networks without wavelength conversion. ETRI Journal, 24(2):131-141, April 2002 .

[12]L. Margada and J. Simon . Wavelength assignment problem on all optical networks with $\mathrm{k}$ fibres per link. In Automata, Langauges and Programming . 27 th International Coloquium, ICALP 2000, volume 1853of Lectures Notes in Computer Cience, pages 768-779, 2000.

[13]K.D.R Assis and H. Waldman “ Topologia Virtual e Topologia Física de Redes Ópticas: Uma Proposta de Projeto Integrado" Revista da Sociedade Brasileira de Telecomunicações SBrT. Vol. 19, n.2, Agosto 2004, pp. 119-126.

[14]K.D.R Assis and H. Waldman , "An Efficient Strategy for the RWA Problem with Allocation of Wavelength Converters", Ghent -Belgium ONDM 04. Ghent Belgium,v.1, february 2004.
[15]R.Dutta and G. Rouskas, "A Survey of Virtual Topology Design Algorithms for Wavelength Routed Optical Networks, “Opt. Net. Mag., "Premiere Issue, " Jan. 2000.

[16]B.Mukherjee, D.Banerjee, S. Ramamurthy, and A.Mukherjee, "Some principles for designing a wide area optical network", IEEE/ACM Trans. Networking, vol. 4, pp. 684-696, Oct. 1996

[17]R. M. Krishnaswamy, K.N. Sivarajan "Design of Logical Topologies: A Linear Formulation for Wavelength-Routed Optical Networks with No Wavelength Changers” IEEE/ACM Transactions on Networking, vol. 9, NO.2, April 2001.

[18]A.M.C.A Koster and Zymolka. "Provably good solutions for wavelength assignment in Optical networks" - Optical Network Design and Modeling- ONDM 2005, Milan, Italy.

[19] Y. Fukushima, H. Harai, S. 'i. Arakawa, and M. Murata, "Design of wavelength-convertible edge nodes in wavelength-routed networks," J. Opt. Netw. 5, 196-209 (2006).

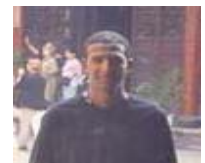

Karcius was born in Feira de Santana-BA, Brazil in 1974. In 1997 he graduated as Electronics Engineer from the Universidade Federal da Paraíba UFPB/Campina Grande-PB, Brazil. In 2000, he received a M.Sc. degree from Universidade Federal do Espírito Santo. He has obtained his Ph.D. degree in July 2004 at the State University of Campinas (UNICAMP). His research activities focused on optical networks and fiber optic digital communication systems. He has written and revised papers on this and related subjects. At the moment Karcius is full professor and researcher of the Federal University of ABC (UFABC).

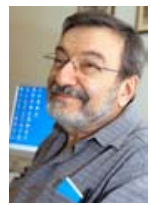

Helio Waldman was born in São Paulo, Brazil, on June 20, 1944. He graduated as Engenheiro de Eletrônica from Instituto Tecnológico de Aeronáutica (ITA) at São José dos Campos, Brazil, in 1966, and received the M.S. and Ph.D. degrees from Stanford University at Stanford, California, USA, in 1968 and 1972, respectively, both in Electrical Engineering. At the moment, Prof. Waldman is with Federal University of ABC (UFABC).

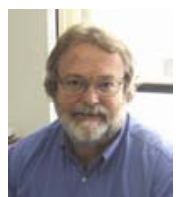

William F. Giozza [M] (giozza@unifacs.br) since 1998, is full professor and researcher at the Computer Networks Group of the Salvador University, in Salvador, Bahia, Brazil. He received his D.Eng. degree in Computer Science from University of Paris, France, in 1982; MSc. in Electrical Engineering from Federal University of Paraíba, Campina Grande, Brazil, in 1979; and B. S. in Electronics Engineering from Aeronautical Technological Institute, São José dos Campos, Brazil, in 1976. From 1977 to 1998, he was professor in Computer Science and Electrical Engineering at Federal University of Paraíba, Campina Grande, Brazil. From 1992 to 1993 he was associate professor at University of Paris 6, France. 
TABLE VI

LIGHTPATHS ESTABLISHED X WAVELENGTH

\begin{tabular}{|c|c|c|c|c|c|c|c|c|c|}
\hline \multicolumn{10}{|c|}{ Number of lightpaths established } \\
\hline \multirow[t]{2}{*}{$W$} & No Conversion & Conversion & \multicolumn{7}{|c|}{ Sparse Conversion } \\
\hline & $N_{c}=0$, for all & $N_{c}=1$, for all & Node & 1 & 2 & 3 & 4 & 5 & 6 \\
\hline \multirow[t]{2}{*}{2} & \multirow[t]{2}{*}{10} & \multirow[t]{2}{*}{11} & $N_{c}$ & 1 & 0 & 0 & 0 & 0 & 0 \\
\hline & & & \multicolumn{7}{|c|}{11} \\
\hline \multirow[t]{2}{*}{3} & \multirow[t]{2}{*}{15} & \multirow[t]{2}{*}{16} & $N_{c}$ & 1 & 0 & 0 & 0 & 0 & 0 \\
\hline & & & \multicolumn{7}{|c|}{16} \\
\hline \multirow[t]{2}{*}{4} & \multirow[t]{2}{*}{20} & \multirow[t]{2}{*}{22} & $N_{c}$ & 1 & 1 & 0 & 0 & 0 & 0 \\
\hline & & & \multicolumn{7}{|c|}{22} \\
\hline \multirow[t]{2}{*}{5} & \multirow[t]{2}{*}{25} & \multirow[t]{2}{*}{27} & $N_{c}$ & 1 & 1 & 0 & 0 & 0 & 0 \\
\hline & & & \multicolumn{7}{|c|}{27} \\
\hline \multirow[t]{2}{*}{6} & \multirow[t]{2}{*}{28} & \multirow[t]{2}{*}{29} & $\overline{N_{c}}$ & 1 & 0 & 0 & 0 & 0 & 0 \\
\hline & & & \multicolumn{7}{|c|}{29} \\
\hline
\end{tabular}

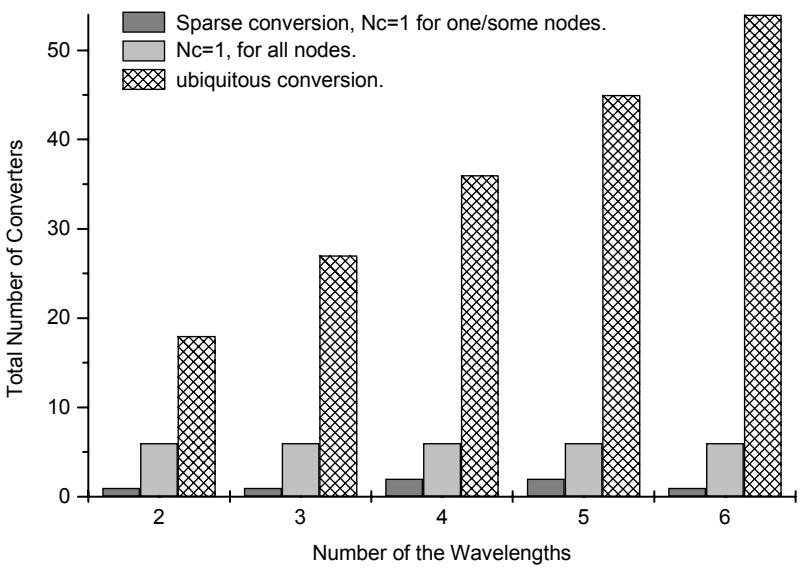

Fig. 22. Results for 6-node mesh Network. Total number of converters $\left(N_{t}\right)$ $N_{t}=\Sigma N_{c}$ for established connections from table III in function of wavelength plan. In ubiquitous conversion, $N_{c}=W . \delta_{p}$, for each node.

TABLE VII

SESSION MATRIX FOR NSFNET (268 CONNECTIONS)

\begin{tabular}{|c|c|c|c|c|c|c|c|c|c|c|c|c|}
\hline 0 & 1 & 3 & 1 & 1 & 1 & 3 & 0 & 2 & 0 & 1 & 2 & 0 \\
\hline 0 & 0 & 0 & 2 & 2 & 2 & 1 & 1 & 1 & 2 & 1 & 0 & 1 \\
\hline 3 & 2 & 0 & 3 & 0 & 1 & 2 & 3 & 1 & 3 & 1 & 2 & 2 \\
\hline 3 & 1 & 0 & 0 & 1 & 1 & 2 & 3 & 2 & 2 & 1 & 2 & 1 \\
\hline 1 & 3 & 0 & 2 & 0 & 1 & 0 & 2 & 0 & 3 & 0 & 1 & 1 \\
\hline 1 & 2 & 1 & 3 & 2 & 0 & 1 & 3 & 3 & 1 & 0 & 1 & 1 \\
\hline 2 & 2 & 3 & 1 & 3 & 3 & 0 & 0 & 3 & 1 & 2 & 0 & 3 \\
\hline 3 & 1 & 2 & 3 & 1 & 0 & 1 & 0 & 0 & 3 & 2 & 0 & 3 \\
\hline 3 & 0 & 1 & 3 & 3 & 3 & 1 & 0 & 0 & 2 & 1 & 1 & 1 \\
\hline 0 & 0 & 0 & 1 & 2 & 0 & 2 & 0 & 1 & 0 & 1 & 0 & 0 \\
\hline 1 & 0 & 0 & 2 & 0 & 3 & 0 & 1 & 0 & 3 & 0 & 3 & 1 \\
\hline 2 & 3 & 1 & 1 & 3 & 2 & 3 & 2 & 2 & 2 & 2 & 0 & 1 \\
\hline 2 & 0 & 1 & 2 & 0 & 1 & 2 & 0 & 3 & 0 & 2 & 1 & 0 \\
\hline 1 & 1 & 0 & 2 & 1 & 0 & 1 & 3 & 0 & 1 & 2 & 1 & 3 \\
\hline
\end{tabular}




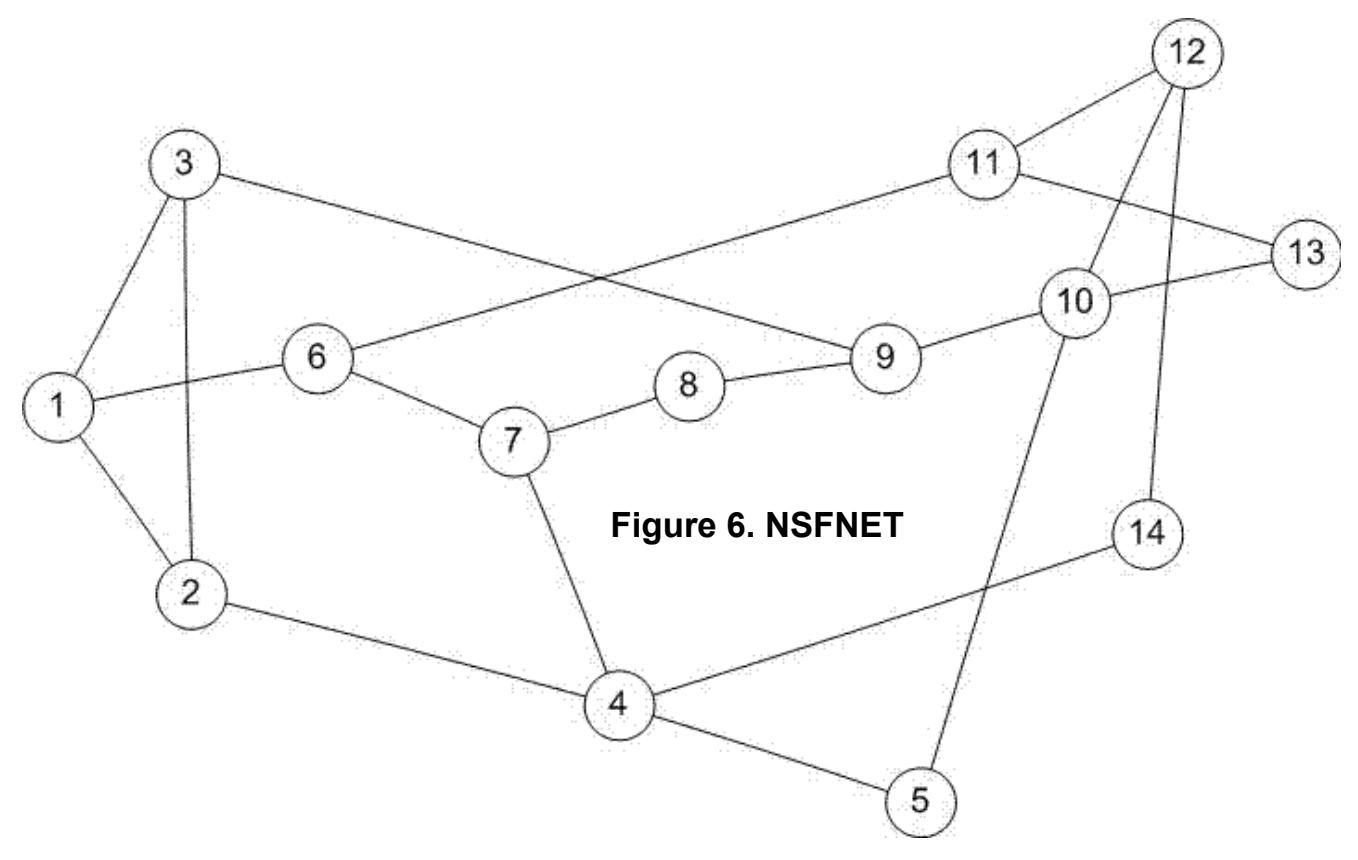

Fig. 23. NSFNET

TABLE VIII

RESULT OBTAINED FOR NSFNET BY LP FORMULATION AND HEURISTICS

\begin{tabular}{|c|c|c|c|c|}
\hline \multirow{2}{*}{$\boldsymbol{W}$} & \multicolumn{4}{|c|}{ Established Connections } \\
\cline { 2 - 5 } & $\begin{array}{c}\text { Upper Bound } \\
\text { (LP) }\end{array}$ & \multicolumn{3}{|c|}{ Heuristic } \\
\cline { 3 - 5 } & & $\mathbf{K S P H}$ & \multicolumn{2}{|c|}{ KSPNc } \\
\cline { 3 - 5 } & $\mathbf{N c}=\mathbf{W} \cdot \boldsymbol{~}_{\boldsymbol{p}}$ & $\mathbf{N c}=\mathbf{7}$ & $\mathbf{N c}=\mathbf{5}$ \\
\hline $\mathbf{1 0}$ & 198 & 187 & 187 & 182 \\
\hline $\mathbf{1 1}$ & 208 & 196 & 196 & 191 \\
\hline $\mathbf{1 2}$ & 218 & 209 & 207 & 203 \\
\hline $\mathbf{1 3}$ & 228 & 220 & 218 & 214 \\
\hline $\mathbf{1 4}$ & 238 & 229 & 227 & 224 \\
\hline $\mathbf{1 5}$ & 248 & 238 & 236 & 233 \\
\hline $\mathbf{1 6}$ & 258 & 246 & 243 & 239 \\
\hline $\mathbf{1 7}$ & 263 & 252 & 247 & 247 \\
\hline $\mathbf{1 8}$ & 267 & 255 & 252 & 251 \\
\hline $\mathbf{1 9}$ & 268 & 258 & 256 & 258 \\
\hline $\mathbf{2 0}$ & 268 & 262 & 259 & 259 \\
\hline $\mathbf{2 1}$ & 268 & 264 & 261 & 260 \\
\hline $\mathbf{2 2}$ & 268 & 266 & 265 & 264 \\
\hline $\mathbf{2 3}$ & 268 & 267 & 267 & 267 \\
\hline $\mathbf{2 4}$ & 268 & 268 & 268 & 268 \\
\hline $\mathbf{2 5}$ & 268 & 268 & 268 & 268 \\
\hline $\mathbf{2 6}$ & 268 & 268 & 268 & 268 \\
\hline & & & & \\
\hline
\end{tabular}

